\section{(6) OPEN ACCESS}

\title{
Dual-energy CT for the diagnosis of gout: an accuracy and diagnostic yield study
}

\author{
Tim Bongartz, ${ }_{1}^{1}$ Katrina N Glazebrook, ${ }^{2}$ Steven J Kavros, ${ }^{3}$ Naveen S Murthy, ${ }^{2}$ \\ Stephen P Merry, ${ }^{4}$ Walter B Franz III, ${ }^{4}$ Clement J Michet, ${ }_{1}{ }^{1}$ Barath M Akkara Veetil, ${ }_{1}^{1}$ \\ John M Davis III, ${ }^{1}$ Thomas G Mason II, ${ }^{1}$ Kenneth J Warrington, ${ }^{1}$ Steven R Ytterberg, \\ Eric L Matteson, ${ }^{1}$ Cynthia S Crowson, ${ }^{5}$ Shuai Leng, ${ }^{2}$ Cynthia H McCollough ${ }^{2}$
}

\begin{abstract}
Handling editor Tore K Kvien
- Additional material is published online only. To view please visit the journal online (http://dx.doi.org/10.1136/ annrheumdis-2013-205095).

${ }^{1}$ Division of Rheumatology, Department of Internal Medicine, Mayo Clinic, Rochester, Minnesota, USA 2Department of Radiology, Mayo Clinic, Rochester, Minnesota, USA

${ }^{3}$ Department of Orthopedic Surgery, Mayo Clinic, Rochester, Minnesota, USA ${ }^{4}$ Department of Family Medicine, Mayo Clinic, Rochester, Minnesota, USA ${ }^{5}$ Division of Biomedical Statistics and Informatics, Department of Health Sciences Research, Mayo Clinic, Rochester, Minnesota, USA
\end{abstract}

\section{Correspondence to} Tim Bongartz, Mayo Clinic College of Medicine, Division of Rheumatology, Department of Internal Medicine, Mayo Clinic, 200 1st Street SW, Rochester, MN 55905, USA; bongartz.tim@mayo.edu

Received 18 December 2013 Revised 17 February 2014 Accepted 1 March 2014 Published Online First 26 March 2014

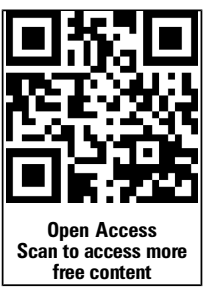

CrossMark

To cite: Bongartz T, Glazebrook KN, Kavros SJ, et al. Ann Rheum Dis 2015;74:1072-1077.

\section{ABSTRACT}

Objectives To assess the accuracy of dual-energy CT (DECT) for diagnosing gout, and to explore whether it can have any impact on clinical decision making beyond the established diagnostic approach using polarising microscopy of synovial fluid (diagnostic yield).

Methods Diagnostic single-centre study of 40 patients with active gout, and 41 individuals with other types of joint disease. Sensitivity and specificity of DECT for diagnosing gout was calculated against a combined reference standard (polarising and electron microscopy of synovial fluid). To explore the diagnostic yield of DECT scanning, a third cohort was assembled consisting of patients with inflammatory arthritis and risk factors for gout who had negative synovial fluid polarising microscopy results. Among these patients, the proportion of subjects with DECT findings indicating a diagnosis of gout was assessed.

Results The sensitivity and specificity of DECT for diagnosing gout was $0.90(95 \% \mathrm{Cl} 0.76$ to 0.97$)$ and $0.83(95 \% \mathrm{Cl} 0.68$ to 0.93$)$, respectively. All false negative patients were observed among patients with acute, recent-onset gout. All false positive patients had advanced knee osteoarthritis. DECT in the diagnostic yield cohort revealed evidence of uric acid deposition in 14 out of 30 patients (46.7\%).

Conclusions DECT provides good diagnostic accuracy for detection of monosodium urate (MSU) deposits in patients with gout. However, sensitivity is lower in patients with recent-onset disease. DECT has a significant impact on clinical decision making when gout is suspected, but polarising microscopy of synovial fluid fails to demonstrate the presence of MSU crystals.

\section{INTRODUCTION}

The incidence of gout has tripled over the recent decades and now represents the most common form of inflammatory arthritis in men and women. ${ }^{1}$ In order to successfully meet the challenge of this surge in disease burden, providers are required to correctly diagnose and distinguish gout from other forms of joint disease.

Currently, the identification of monosodium urate (MSU) crystals in synovial fluid (SF) or tissue aspirates is the only certain way to diagnose gout. Unfortunately, the high dependency on microscopic analyses of biospecimens is not without problems: obtaining adequate specimens from small joints or periarticular structures can be difficult especially in the primary care setting, where the majority of patients with gout are seen. ${ }^{2}$ In addition to the challenges associated with sampling of appropriate SF specimens, the reliability of polarising microscopy is poor. ${ }^{3} 4$

Since a diagnosis of gouty arthritis typically results in therapeutic steps that are distinctly different from those used to address other types of inflammatory arthritis, the failure to detect MSU deposition can result in exposure to unnecessary and ineffective treatment strategies. Alternative tests for the detection of MSU crystals, which could aid clinicians in challenging diagnostic situations, would therefore be desirable.

Dual-energy CT (DECT) has been found to be highly accurate in detecting and classifying uric acid renal stones. ${ }^{5}$ The unique chemical composition of uric acid precipitates results in a distinct radiographic attenuation when compared with other materials. This results in characteristic patterns of CT numbers at high versus low kilovolts $(\mathrm{kV})$-allowing imaging software to differentiate uric acid stones from other types of stones.

While some studies have explored the use of DECT for articular MSU detection in patients with long-standing and tophaceous gout, ${ }^{8}{ }^{9}$ no formal assessment of tests characteristic across the whole spectrum of acute gouty arthritis, including patients with early disease, has been performed. Therefore, we aimed to investigate the accuracy of DECT for diagnosing gout in a prospective study across a wide spectrum of disease manifestations. Additionally, we explored the question if DECT would affect clinical decision making beyond the current routine diagnostic approach.

\section{METHODS \\ Accuracy study \\ Study subjects}

For study participation, we screened patients who presented to the Mayo Clinic Rochester Rheumatology procedure clinic with joint pain and/or swelling. All patients were scheduled to undergo a clinically indicated injection and/or aspiration of a peripheral joint (small finger joint, wrist, elbow, knee, small foot joint or ankle). Patients with tophaceous gout were excluded. After written informed consent was provided, we aspirated SF from a symptomatic joint. If patients were referred for aspiration/injection of more 
than one joint, the clinically most prominently involved joint was chosen as the index joint.

We assessed the adequacy of each sample based on fluid volume (at least a drop), consistency (viscous) and presence of lymphocytes (at least 10 lymphocytes per visual field). Patients with an inadequate sample were excluded.

Based on the results of our reference test for the presence of MSU crystals, patients were divided into two groups: patients with gout versus control subjects with other types of joint disease. Accrual of patients with gout was stratified according to disease duration: we aimed to include at least $50 \%$ of patients with no prior history of gout and symptom duration $<6$ weeks.

The study was conducted according to the ethical principles of the Declaration of Helsinki and approved by the Mayo Clinic Institutional Review Board (IRB).

\section{Reference test}

Demonstration of MSU crystals in SF is generally regarded as the most specific, though not very sensitive, method to make a diagnosis of gout. ${ }^{10}$ For the purpose of our study, reference testing of SF was performed on all adequate samples: two rheumatologists who were blinded to each other's assessment, performed polarising microscopy examination (Zeiss polarising microscope, Jena, Germany) within minutes of sample acquisition. In advance of the study, the investigators who performed polarising microscopy had to correctly identify 10 test specimens prior to the study (5 cases of gout, 5 control cases).

Presence of intracellular and/or extracellular needle-shaped, negatively birefringent crystals was consistent with a diagnosis of gout. ${ }^{11}$ In case of divergent polarising microscopy results, the specimen was reviewed by a third investigator who made the final determination regarding the presence of MSU crystals.

In order to increase sensitivity of our reference standard, electron microscopy was performed on all samples for detection of needle-shaped crystalline material. The sample was prepared by placing $1 \mu \mathrm{L}$ of SF onto a parlodian carbon-coated 300 mesh titanium grid and allowed to dry. The grid was examined for evidence of gout crystals with a Field Emission Inc (FEI) Tecnai 12 transmission electron microscope operating at $80 \mathrm{kV}$. Elemental composition of crystals was determined by analysis of energy-dispersive spectra collected by an EDAX X-ray microanalysis system. Patients were diagnosed with gout if either polarising microscopy or electron microscopy, or both, confirmed the presence of MSU crystals.

\section{Test under study}

A CT examination of the aspirated joint area was performed using a dual-source CT scanner operating in DECT mode within 2 weeks of joint aspiration. Depending on the location of the index joint, we imaged one of four different joint areas: hand/ wrist, elbow, knee or foot/ankle. For example, when a patient presented with acute arthritis of the right first metatarsophalangeal (MTP) joint, the right foot and ankle were imaged. The technical details of our imaging method have been described elsewhere. ${ }^{9}$ In brief, patients were examined with a dual-source CT scanner operated at DECT mode (SOMATOM Definition Flash, Siemens Medical Systems, Forchheim, Germany). The tube potentials were 80 (tube B) and $140 \mathrm{kV}$ (tube A) with an additional tin filter. The use of a different $\mathrm{kV}$ for each tube exploits the $\mathrm{kV}$-dependent nature of CT numbers, allowing differentiation of materials having different effective atomic numbers. Post-processing was performed using a commercial software programme ('Gout', Syngo CT Workplace, Siemens Medical Systems) to create material-selective images, where
MSU deposits were colour-coded as green. For detailed DECT settings, please see online supplement 1 .

\section{Analysis}

A musculoskeletal radiologist (KNG or NSM), who was aware of the location of the index joint but blinded to the patient's microscopy results, evaluated the images using a commercially available workstation. Axial images, as well as images reconstructed in the sagittal/coronal planes and volume-rendered three-dimensional views every $24^{\circ}$ around the imaged joint, were reviewed. The presence of green-coloured voxels (independent of the number of voxels) in articular or periarticular structures of the index joint was classified as positive for the presence of MSU crystals. Of note, green pixilation in joint areas other than the index joint did not constitute a positive test result for our primary analysis. The sensitivity and specificity (95\% CI) of DECT scanning for the detection of MSU deposition were calculated. In a secondary analysis, we tested the hypothesis if classifying DECT as 'positive' even when green pixilation was absent in the index joint but present in other areas would result in higher test accuracy.

\section{Diagnostic yield study}

Study subjects

To explore the contribution of DECT to clinical decision making after routine diagnostic testing for gout has been exhausted, we assembled a separate cohort of patients: in these subjects, the treating physician had considered gout as an important differential diagnosis, but an appropriate SF specimen for analysis could either not be obtained because of technical difficulties, or polarised microscopy was negative for the presence of MSU crystals. Additionally, patients had to meet at least one of the following criteria which are associated with an increased pretest likelihood of gout ${ }^{10}$ : history or presence of podagra, serum uric acid $\geq 7 \mathrm{mg} / \mathrm{dL}$ in men or $\geq 6 \mathrm{mg} / \mathrm{dL}$ in women, asymmetrical joint swelling, radiographic subcortical cyst, erythema and paroxysmal character of complaints (rapid development of pain that reaches its maximum in 6-12 h). We excluded patients with a known history of gout or presence of tophi.

\section{Imaging and diagnostic confirmation}

All patients underwent DECT imaging of the affected joint area (for technical details, see above). Images were read by a musculoskeletal radiologist. If DECT images revealed green pixilation in or around the clinically affected joint area, we performed an ultrasound-guided aspiration of these areas with subsequent polarising microscopy. The procedure was performed by an experienced musculoskeletal sonographer (ELM or TB) who reviewed the DECT scan prior to the procedure to better target areas of MSU deposition. We performed polarising microscopy of the aspirate with two observers who were blinded to each other's assessment. We calculated the frequency of MSU crystal deposition detected with DECT scanning, and the proportion of patients where presence of MSU deposits could be confirmed through image-guided aspiration.

\section{RESULTS}

\section{Accuracy study}

Flow of subjects and clinical characteristics

Between October 2010 and September 2012, 119 consecutive patients referred for a joint aspiration and/or injection were screened for study participation. Of these, 28 patients were excluded as screen failures because of inadequate SF samples. 


\begin{tabular}{|c|c|c|}
\hline & $\begin{array}{l}\text { Patients with } \\
\text { gout }(n=43)\end{array}$ & $\begin{array}{l}\text { Patients without } \\
\text { gout }(n=48)\end{array}$ \\
\hline Age, mean (SD), years & $62.1(13.2)$ & $58.7(13.7)$ \\
\hline \multicolumn{3}{|l|}{ Sex } \\
\hline Male & $34(79)$ & $19(40)$ \\
\hline Female & $9(21)$ & $29(60)$ \\
\hline \multicolumn{3}{|l|}{ Index joint } \\
\hline MCP/PIP & $3(7)$ & $4(8)$ \\
\hline Wrist & $2(5)$ & $2(4)$ \\
\hline Elbow & $1(2)$ & $0(0)$ \\
\hline MTP & $26(60)$ & $18(38)$ \\
\hline Mid-foot/ankle & $9(21)$ & $9(19)$ \\
\hline Knee & $2(5)$ & $15(31)$ \\
\hline Symptom duration (week) & & NA \\
\hline$\leq 6$ & $29(67)$ & - \\
\hline$>6$ & $14(33)$ & - \\
\hline Prior history of gout & $15(35)$ & NA \\
\hline Serum UA, mean (SD), mg/dL & $8.0(2.0)$ & NA \\
\hline \multicolumn{3}{|l|}{ Referral diagnosis } \\
\hline Gout & $40(93)$ & $2(4)$ \\
\hline DJD & - & $21(44)$ \\
\hline RA & - & $12(25)$ \\
\hline Septic joint & - & $1(2)$ \\
\hline CTD & - & $1(2)$ \\
\hline CPPD & $1(2)$ & $3(6)$ \\
\hline Unknown & $2(5)$ & $7(15)$ \\
\hline \multicolumn{3}{|c|}{$\begin{array}{l}\text { Except where stated otherwise, values are the number (\%) of patients. } \\
\text { CPPD, calcium pyrophosphate deposition disease; CTD, connective tissue disease; } \\
\text { DJD, degenerative joint disease; MCP, metacarpophalangeal joint; MTP, } \\
\text { metatarsophalangeal joint; n, number; PIP, proximal interphalangeal joint; RA, } \\
\text { rheumatoid arthritis; UA, uric acid. }\end{array}$} \\
\hline
\end{tabular}

Gout was diagnosed in 43 patients, based on our combined reference method using polarising and electron microscopy; 20 presented with a first flare of inflammatory arthritis and a symptom duration $<6$ weeks. The majority of patients with gout had small joint arthritis $(n=29)$, mainly involving the MTP or metacarpophalangeal joints. Fourteen patients had medium or large joint arthritis, most commonly affecting the wrist or ankle. The control cohort consisted of 48 patients who each had a SF analysis that was negative for MSU crystals. Twenty-two patients had small joint and 26 patients had medium or large joint disease. For detailed patient characteristics, see table 1 .

Of those patients, three with gout and seven controls did not return for their DECT scan, leaving 40 in the gout group and 41 in the control group for final analysis (figure 1). The level of agreement between the two observers performing polarising microscopy was high: out of 91 specimens, only three were assessed differently and final adjudication had to be performed by a third observer $(\kappa=0.93,95 \%$ CI 0.86 to 1.0$)$.

\section{Results for DECT}

In the 40 patients with gout who underwent DECT, imaging demonstrated MSU deposition (figure 2A) around the index joint in 36 patients (90.0\%). All four cases of gout that were missed with DECT (false negatives) occurred among patients with a first flare of gout and symptom duration $<6$ weeks. Among the 41 control patients in whom SF analysis was negative, DECT demonstrated evidence of MSU deposition in seven cases. All these cases occurred in patients with knee osteoarthritis (OA), and DECT imaging results indicated MSU deposition within the cartilage/menisci (figure 2B).

Based on these results, the overall sensitivity and specificity of DECT for diagnosing gout was 0.90 (95\% CI 0.76 to 0.97$)$ and

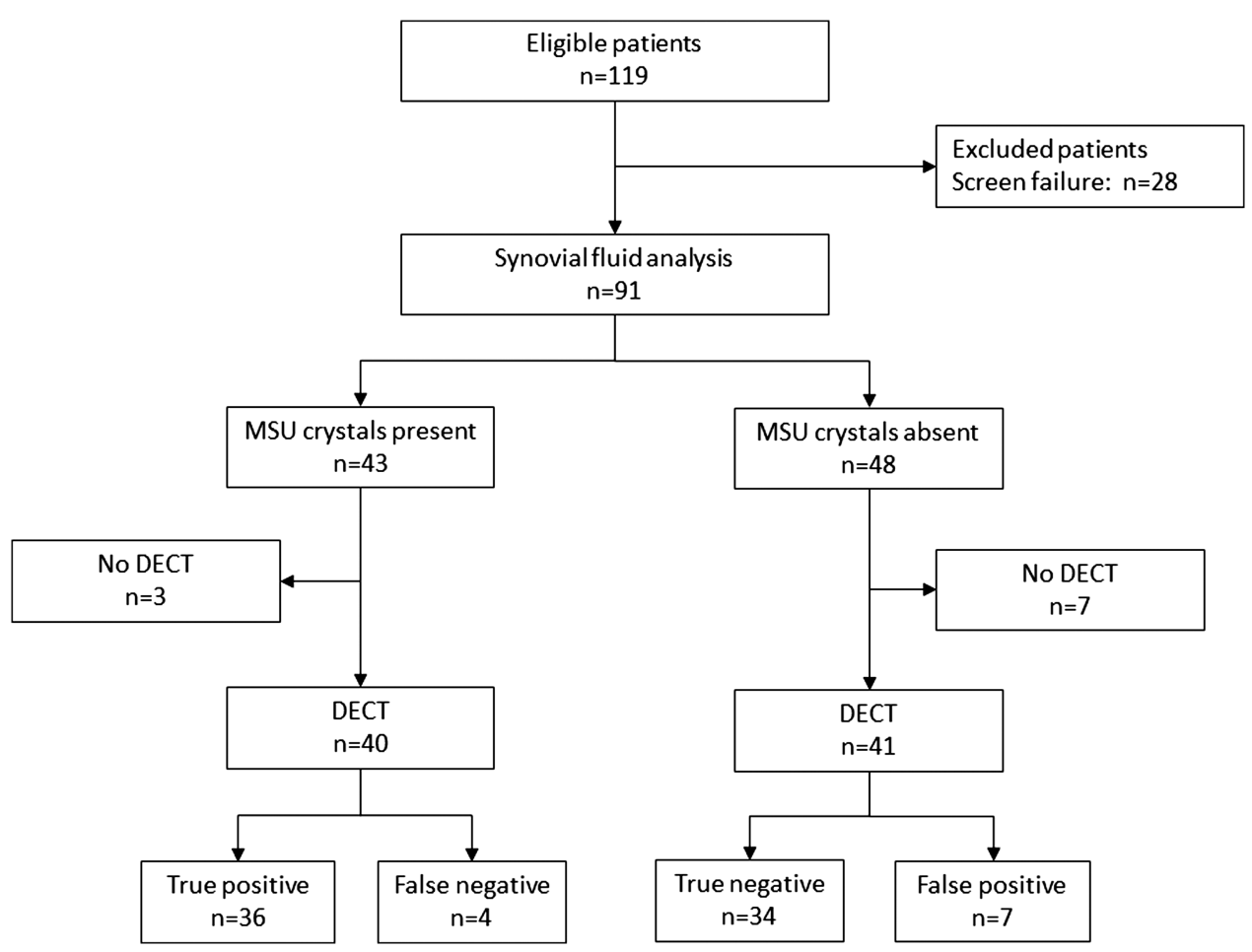

Figure 1 Patient flow, diagnostic accuracy study. DECT, dual-energy computer tomography; MSU, monosodium urate. 
0.83 (95\% CI 0.68 to 0.93 ), respectively. No adverse events due to the use of DECT, or as a result of SF aspiration, occurred. For the secondary analyses, we classified imaging results as positive for gout if green pixilation was either observed around the index joint and/or in other locations of the imaged area. Two of the four patients with a first flare of gouty arthritis but no imaging findings of MSU deposition in the index joint had green pixilation in other areas. Conversely, 18 control patients had areas of green pixilation beyond the symptomatic joint. These results translate into a higher sensitivity of 0.95 (95\% CI 0.83 to 0.99$)$, but a significant drop in specificity to $0.56(95 \%$ CI 0.40 to 0.72 ).

\section{Diagnostic yield study}

Study subjects

Thirty patients with inflammatory arthritis or tendinitis, who either had a negative SF analysis $(n=22)$ or were thought not to be good candidates for SF aspiration $(n=8)$, were included in our diagnostic yield cohort. For detailed patient characteristics, see table 2 .

\section{DECT results}

DECT demonstrated green pixilation in or around the clinically involved joint in 14 of the 30 patients, suggestive for MSU deposition. Importantly, MSU deposits were located in periarticular structures of the index joint (tendon/tendon insertion sites/entheses) in a majority of these patients (figure 2C,D).

Confirmation of DECT findings through ultrasound-guided aspiration

Ultrasound-guided aspiration of the area was performed in 12 of the 14 patients in whom green pixilation was detected by DECT (figure 2C,D). Two patients declined confirmatory aspiration. MSU crystals were found on polarising microscopy in 11 of the 12 patients. In one patient with DECT images indicating MSU deposition in the knee, we were unable to confirm MSU deposition on examination of the SF.

Overall, DECT helped to diagnose gout in 11 out of 30 patients with undifferentiated arthritis/tendinitis/enthesitis who had a negative polarising microscopy result from their initial diagnostic evaluation (figure 3).

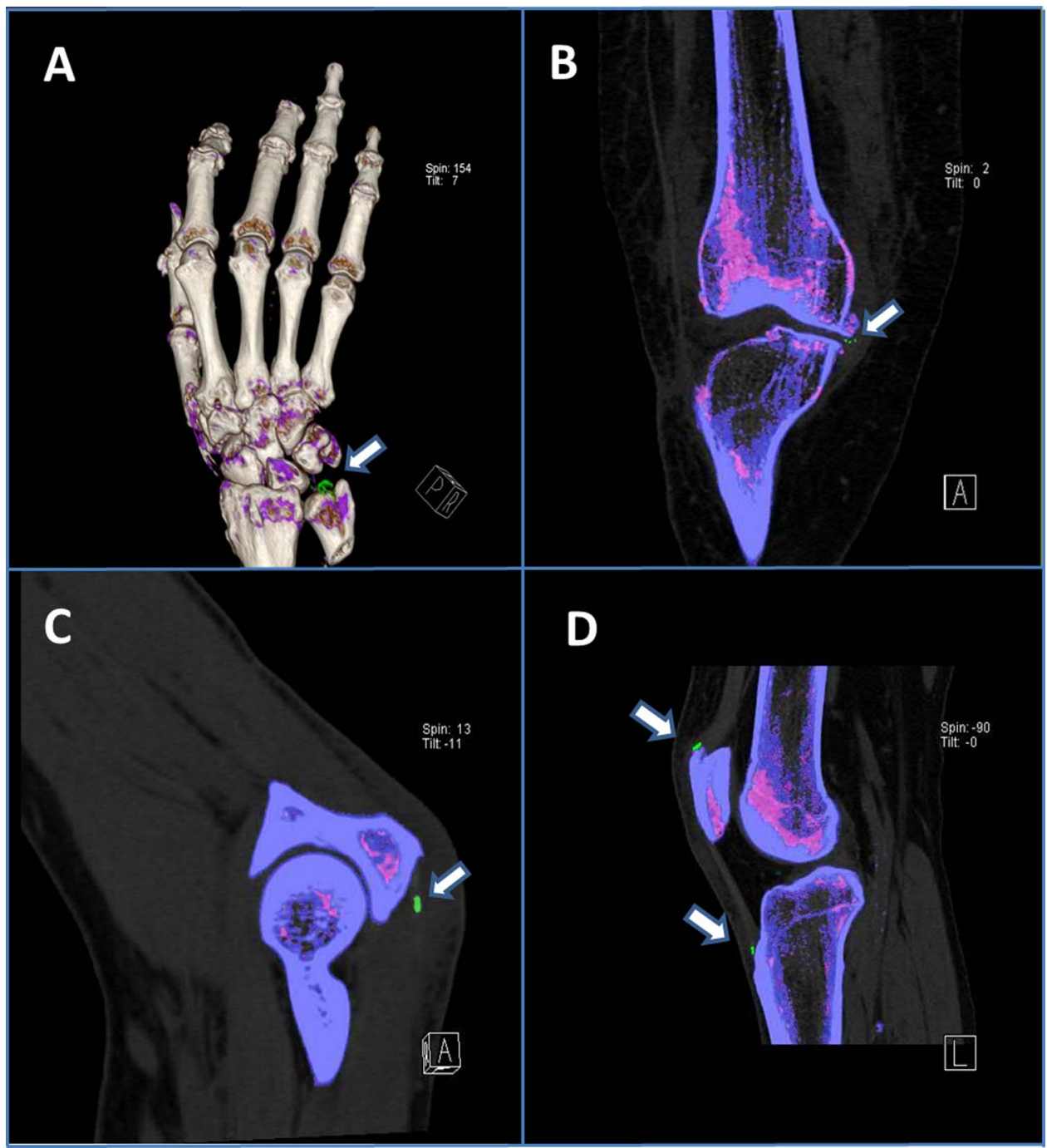

Figure 2 Dual-energy CT images. Arrows indicate MSU deposition (MSU deposits colour coded in green). (A) Subject accuracy study with acute gouty arthritis of the wrist (three-dimensional (3D) reconstruction image); (B) Subject accuracy study with advanced osteoarthritis of the knee, synovial fluid analysis negative for MSU crystals; (C) Subject diagnostic yield study with elbow pain and swelling, synovial fluid analysis negative for MSU crystals; (D) Subject diagnostic yield study with knee pain and swelling, synovial fluid analysis negative for MSU crystals. MSU, monosodium urate. 


\begin{tabular}{|c|c|}
\hline & $\begin{array}{l}\text { Patients with clinical } \\
\text { suspicion for gout }(n=30)\end{array}$ \\
\hline Age, mean $(S D)$, years & $64.7(12.9)$ \\
\hline \multicolumn{2}{|l|}{ Sex } \\
\hline Male & $24(80)$ \\
\hline Female & $6(20)$ \\
\hline \multicolumn{2}{|l|}{ Symptomatic joint area } \\
\hline Toe & $4(13)$ \\
\hline Foot & $8(27)$ \\
\hline Ankle & $4(13)$ \\
\hline Knee & $3(10)$ \\
\hline Finger & $2(7)$ \\
\hline Wrist & $8(27)$ \\
\hline Elbow & $1(3)$ \\
\hline \multicolumn{2}{|l|}{ Symptom duration } \\
\hline$\leq 1$ month & $7(23)$ \\
\hline$>1$ month, $<3$ months & $9(30)$ \\
\hline$\geq 3$ months & $14(47)$ \\
\hline \multicolumn{2}{|c|}{ Factors associated with an increased risk of gout } \\
\hline Previous episode of podagra & $12(40)$ \\
\hline Serum uric acid increased ${ }^{*}(n=29)$ & $19(63)$ \\
\hline Asymmetric swelling & $22(73)$ \\
\hline Erythema & $15(50)$ \\
\hline Subcortical cyst & $6(20)$ \\
\hline Paroxysmal onset of complaints & $14(47)$ \\
\hline Joint aspiration negative for MSU crystals & $22(73)$ \\
\hline Joint aspiration not performed & $8(27)$ \\
\hline \multicolumn{2}{|c|}{ Reasons for not performing joint aspirations $(n=8)$} \\
\hline Patient declined & $1(3)$ \\
\hline Possible cellulitis & $2(7)$ \\
\hline No effusion & $5(17)$ \\
\hline
\end{tabular}

\section{DISCUSSION}

The present study confirms the good overall accuracy of DECT in diagnosing patients with gout. Moreover, DECT can provide important diagnostic information when the current routine diagnostic approach has failed to confirm the presence of MSU crystals. Notwithstanding these results, our data underscore the importance of careful patient selection before using DECT as a diagnostic modality, as false positive/false negative results were seen in two particular clinical scenarios: First, DECT appears to have limited sensitivity in patients with acute gout and no prior episodes of gouty arthritis. Twenty per cent of patients with early disease did not demonstrate evidence of MSU deposition on DECT images. It appears likely that small MSU deposits in patients early during the disease process may remain undetected.

Second, DECT may have limited specificity in knee OA. In our control group, DECT revealed green pixilation suggestive of MSU deposition in the articular cartilage in a significant proportion of patients, who all had Kellgren-Lawrence grade 3 or 4 OA. None of these patients had evidence of MSU crystals in their SF or a history of gout. It is unclear whether these deposits represent an imaging artefact or if they indicate intracartilaginous MSU deposition that may occur in the context of cartilage damage-associated matrix exposure, and subsequent MSU crystallisation. $^{12} 13$ Importantly, it remains to be seen whether less advanced OA may also demonstrate similar evidence of intracartilaginous uric acid deposition. Of all patients in the control group, only two had lower than grade 3 or 4 radiographic changes of their knee, prohibiting any meaningful conclusion regarding the occurrence of artefact/subclinical uric acid deposition in the knee that is not affected by advanced OA.

DECT enables detection of MSU deposition in anatomic structures that cannot be easily aspirated. Therefore, it is important to reach consensus on what constitutes a 'positive' DECT result. In our study, we have defined 'positive DECT' as detection of MSU deposits in the symptomatic joint area. Our secondary analysis revealed a significant drop in specificity when classifying DECT images as positive for gout when green pixilation was present only in anatomic areas beyond the clinically affected joint, but not around the index joint itself.

Previous studies and case series that have explored the test characteristics of DECT in patients with gout have mainly included subjects with long-standing and/or tophaceous disease-a clinical situation where detection of MSU deposition by DECT scanning is usually not required for diagnostic confirmation. ${ }^{8} 1415$ Since the performance of a diagnostic test is easily overestimated when it is used to evaluate patients at the extremes of the disease spectrum, we excluded patients with tophi from our study. The lower sensitivity of DECT in patients with a first gouty attack underscores that accuracy estimates based on patients with long-standing tophaceous gout should not be generalised across the whole spectrum of disease.

In our diagnostic yield cohort, DECT correctly identified several individuals with gout who had a previous negative SF analysis. The high proportion (approx 30\%) of patients that were missed with the routine diagnostic method is noteworthy. Interestingly, in several patients in whom SF aspiration was negative, DECT demonstrated MSU deposits around tendon and
Figure 3 Patient flow, diagnostic yield study. DECT, dual-energy computer tomography; US, ultrasound.

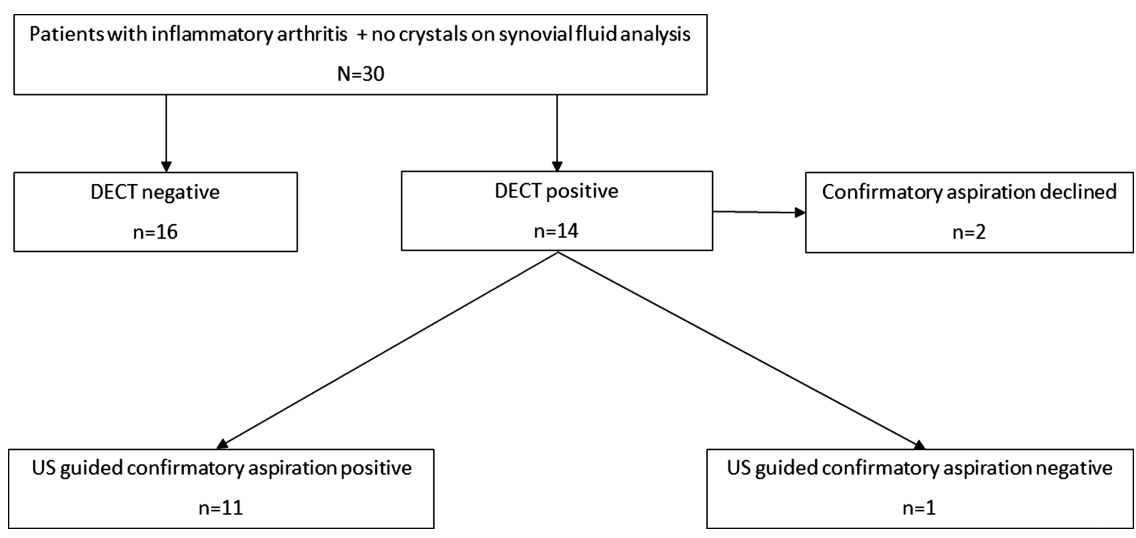

Bongartz T, et al. Ann Rheum Dis 2015;74:1072-1077. doi:10.1136/annrheumdis-2013-205095 
ligament attachment sites. These extra-articular deposits may explain why analysis of intra-articular SF did not reveal presence of MSU crystals.

Our study does have some limitations. Our data indicate that test accuracy is likely to differ between different anatomic areas, as reflected by the low specificity of DECT in osteoarthritic knees. Therefore, our overall accuracy estimate may not apply to every joint area.

Additionally, gout can affect joint areas which were not part of our study, or were only represented in very low numbers, such as the spine, the shoulders and the elbows. ${ }^{16}$ Therefore, the performance of DECT for these joint areas is unclear and images should be interpreted with caution. Interestingly, in several patients with an unremarkable SF analysis, DECT did demonstrate evidence of MSU deposition in tendon sheaths and entheseal sites. This observation suggests that gouty tendinitis and enthesitis are important manifestations of gout that can be difficult to confirm given the challenges of obtaining appropriate specimens from these anatomical sites.

The results of our diagnostic yield study suggest that in a significant number of patients, the diagnosis of gout may be missed when relying on SF analysis alone. Individual skills of the proceduralist who is performing the SF aspiration, the quality of the polarising microscopy, and availability of equipment, can all impact on the likelihood of detecting MSU crystals. ${ }^{17}$ As a result, the overall diagnostic yield will vary among centres, and our single-centre experience may not be representative for other institutions.

Based upon our results, the current application for DECT appears to be the second-line diagnostic evaluation of patients with possible gouty arthritis in whom polarising microscopy of SF has failed to confirm a diagnosis. Future studies will have to clarify how the use of DECT in this clinical situation compares with the use of musculoskeletal ultrasound, which may offer a very similar test performance at lower costs. ${ }^{18}$ DECT can be a highly valuable diagnostic tool in this patient group, helping to avoid unnecessary testing for other inflammatory joint disorders and treatment errors when the diagnosis of gout is missed.

Acknowledgements We would like to thank Jon E Charlesworth for optimising and applying electron microscopy for urate detection.

Contributors All authors contributed and finally approved the current manuscript.

Funding John M Nasseff Clinician Scholarship Award.

Competing interests Siemens Medical Solutions provided software to be used on one of the systems involved in the study. Siemens also provided partial salary support for CHM through an unrestricted research grant to the CT Innovation Center, Mayo Clinic, Rochester, Minnesota, USA. KNG received a travel grant from Siemens Medical Solutions.

Ethics approval The study was approved by the Mayo Clinic Institutional Review Board.
Data sharing statement The copyright transfer agreement is subject to applicable Mayo terms located on the following page: (http://www.mayo.edu/copyright/).

Provenance and peer review Not commissioned; externally peer reviewed.

Open Access This is an Open Access article distributed in accordance with the Creative Commons Attribution Non Commercial (CC BY-NC 3.0) license, which permits others to distribute, remix, adapt, build upon this work non-commercially, and license their derivative works on different terms, provided the original work is properly cited and the use is non-commercial. See: http://creativecommons.org/ licenses/by-nc/3.0/

\section{REFERENCES}

1 Zhu Y, Pandya BJ, Choi HK. Prevalence of gout and hyperuricemia in the US general population: the National Health and Nutrition Examination Survey 20072008. Arthritis Rheum 2011;63:3136-41.

2 Pal B, Foxall M, Dysart $\mathrm{T}$, et al. How is gout managed in primary care? A review of current practice and proposed guidelines. Clin Rheumatol 2000;19:21-5.

3 Segal JB, Albert D. Diagnosis of crystal-induced arthritis by synovial fluid examination for crystals: lessons from an imperfect test. Arthritis Care Res 1999;12:376-80.

4 von Essen R, Holtta AM, Pikkarainen R. Quality control of synovial fluid crystal identification. Ann Rheum Dis 1998;57:107-9.

5 Graser A, Johnson TR, Bader M, et al. Dual energy CT characterization of urinary calculi: initial in vitro and clinical experience. Invest Radiol 2008;43: 112-19.

6 Primak AN, Fletcher JG, Vrtiska TJ, et al. Noninvasive differentiation of uric acid versus non-uric acid kidney stones using dual-energy CT. Acad Radiol 2007; 14:1441-7.

7 Johnson TR, Krauss B, Sedlmair M, et al. Material differentiation by dual energy CT: initial experience. Eur Radiol 2007;17:1510-17.

8 Choi HK, Burns LC, Shojania K, et al. Dual energy CT in gout: a prospective validation study. Ann Rheum Dis 2012;71:1466-71.

9 Glazebrook KN, Guimaraes LS, Murthy NS, et al. Identification of intraarticular and periarticular uric acid crystals with dual-energy CT: initial evaluation. Radiology 2011;261:516-24.

10 Zhang W, Doherty M, Pascual E, et al. EULAR evidence based recommendations for gout. Part I: Diagnosis. Report of a task force of the Standing Committee for International Clinical Studies Including Therapeutics (ESCISIT). Ann Rheum Dis 2006;65:1301-11.

11 Wallace SL, Robinson H, Masi AT, et al. Preliminary criteria for the classification of the acute arthritis of primary gout. Arthritis Rheum 1977;20:895-900.

12 Denoble AE, Huffman KM, Stabler TV, et al. Uric acid is a danger signal of increasing risk for osteoarthritis through inflammasome activation. Proc Natl Acad Sci USA 2011;108:2088-93.

13 Nowatzky J, Howard R, Pillinger $\mathrm{MH}$, et al. The role of uric acid and other crystals in osteoarthritis. Curr Rheumatol Rep 2010;12:142-8.

14 Choi HK, Al-Arfaj AM, Eftekhari A, et al. Dual energy computed tomography in tophaceous gout. Ann Rheum Dis 2009;68:1609-12.

15 Desai MA, Peterson JJ, Garner HW, et al. Clinical utility of dual-energy CT for evaluation of tophaceous gout. Radiographics 2011;31:1365-75; discussion 13761367.

16 Parikh $\mathrm{P}$, Butendieck $\mathrm{R}$, Kransdorf $\mathrm{M}$, et al. Detection of lumbar facet joint gouty arthritis using dual-energy computed tomography. J Rheumatol 2010;37: 2190-1.

17 Swan A, Amer H, Dieppe P. The value of synovial fluid assays in the diagnosis of joint disease: a literature survey. Ann Rheum Dis 2002;61:493-8.

18 Gruber M, Bodner G, Rath E, et al. Dual-energy computed tomography compared with ultrasound in the diagnosis of gout. Rheumatology (Oxford) 2014;53:173-9. 